\title{
Stability of liquid bridges between twisted elliptical disks
}

\author{
A. Laveron-Simavilla , E. Checa , J.M. Perales \\ IDRIUPM, E.T.S.I. Aeronáuticos, Universidad Politécnica de Madrid. 28040 Madrid. Spain \\ ESA-ESTEC, P.O. Box 299. 2200 AG Noordwijk, The Netherlands
}

\begin{abstract}
The influence in the stability of long liquid bridges supported between two elliptical-shaped disks of their main axis relative orientation is investigated. A numerical continuation method capable of finding equilibrium shapes, both stable and unstable, is used to calculate a series of equilibrium shapes supported by disks of increasing eccentricity for different relative orientation of the disks axis. The stable or unstable character of each of the shapes is calculated to determine the position of the stability limit and its character.
\end{abstract}

Keywords: Liquid bridge; Capillarity; Microgravity

\section{Introduction}

A liquid bridge is a model widely used to study the fluid configuration present in the crystal growth technique. The first configuration studied in literature was a cylindrical shape held between two parallel, coaxial circular disks of the same diameter. The response of the mentioned configuration subjected to various disturbances has been studied, including the calculation of the equilibrium shapes and their stability limits (Slobozhanin and Perales, 1993; Slobozhanin and Alexander, 1998; Lowry and Steen, 1997; Marr-Lyon et al., 1997; González and Castellanos, 1993; Mahajan et al., 1999; Parra et al., 2002; Luengo et al., 2003). The influence of different perturbations on the stability of liquid bridges has been extensively analyzed from both the theoretical and the experimental point of view.

Leaving apart non-mechanical disturbances, some works dealing with non-axisymmetric supporting disks have been published, two of them dealing with the equilibrium shapes of liquid bridges between non-circular supports (Martínez et al., 2002a,b), one dealing with the asymptotic stability of long liquid bridges between noncircular disks (Meseguer et al., 2001) and another in which the stability and the cquilibrium shapes of a liquid bridge held between a circular and an clliptic disk is studied (Laverón-Simavilla et al., 2003). In the last two papers mentioned it is demonstrated that the influence on the stability limit of perturbations like an axial gravity and the existence of noncircular supporting disks can interact in the way of balancing each other. The destabilizing effect of an axial gravity can be counteracted by placing the liquid column between noncircular disks. The present paper studies a liquid bridge configuration held between two parallel and coaxial clliptic disks, analyzing the influence of the angle formed between the main axes of the disks, the excentricity of the cllipses and a gravity parallel to the disks. The stability limits and the equilibrium shapes of the configuration are calculated using a numerical method already implemented for analyzing stability problems of non-axisymmetric liquid bridges held between circular disks 
(Laverón-Simavilla and Perales, 1995; Laverón-Simavilla and Checa, 1997) and here adapted to the particularities imposed by the non-axisymmetric boundary conditions.

\section{Formulation}

The fluid configuration considered consists of a mass of liquid of volume $\hat{V}$ held by surface tension forces between two parallel, coaxial, elliptical disks placed a distance $L$ apart. The disks' bigger and smaller semi-axes equal to $R_{0}(1+a)$ and $R_{0}(1-a)$, respectively, and the directions defined by the two disks' bigger axes form an angle $\phi$. The liquid bridge is subjected to an acceleration $\vec{g}=g \vec{u}$, being $\varphi$ the angle formed by the lower disk's bigger main axis and the direction of the acceleration, and $\vec{u}$ the unit vector in the direction of the acceleration as sketched in Fig. 1.

The equation governing the steady shape of the liquid bridge is obtained by expressing the equilibrium between the surface tension forces and the local pressure forces at the free surface of the liquid. This equation can be written using dimensional parameters and variables, as follows:

$\sigma \tilde{M}(\tilde{F})+\tilde{P}-\Delta \rho g \tilde{F} \cos \theta=0$,

where $\vec{M}(\tilde{F})$ is twice the mean curvature of the interface, $\tilde{F}=\vec{F}(\tilde{z}, \theta)$ is the equation for the liquid bridge interface, $\Delta \rho$ the density difference between the liquid bridge and the outer bath, $\sigma$ the surface tension, and $\tilde{P}$ is a constant related to the origin of pressure. The boundary conditions and constraints express the azimuthal periodicity of the interface shape, $\tilde{F}(\tilde{z}, \theta)=\tilde{F}(\vec{z}, \theta+2 \pi)$, the constant value of the liquid bridge volume, and that the bridge surface is anchored or pinned to the edges of the supporting disks.

Let, in a general case, $\tilde{F}( \pm L / 2, \theta)$ be the shape of the supporting disks. If the following characteristic length, associated to the disk shape, is introduced:

$R=\frac{1}{2 \pi} \int_{0}^{2 \pi} \tilde{F}( \pm L / 2, \theta) \mathrm{d} \theta$

then, taking $R$ as characteristic length of the problem, and defining $F=\tilde{F} / R, z=\bar{z} / R$, Eq. (1) can be written as

$M(F)+P-B_{1} F \cos \theta=0$,

where $\tilde{P}$ has been made dimensionless using $\sigma / R, B_{1}$ is the lateral Bond number, $B_{1}=\Delta \rho g R^{2} / \sigma$, and

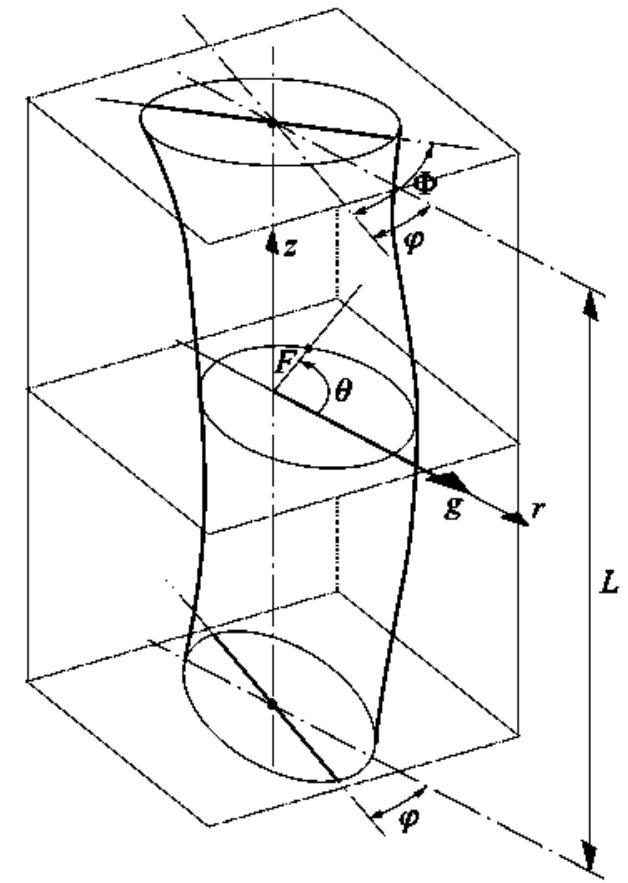

Fig. 1. Geometry and coordinate system for the liquid bridge configuration considered.

is twice the dimensionless mean curvature. The subscripts $z$ and $\theta$ indicate derivatives with respect to these variables.

For the disk configuration considered in this paper, the boundary conditions expressing that the bridge surface is anchored or pinned to the edges of the supporting disks are:

$$
\begin{aligned}
& \tilde{F}(\tilde{z}=-L / 2, \theta)=\frac{R_{0}\left(1-a^{2}\right)}{\left(1+a^{2}-2 a \cos 2(\theta+\varphi)\right)^{1 / 2}}, \\
& \tilde{F}(\tilde{z}=L / 2, \theta)=\frac{R_{0}\left(1-a^{2}\right)}{\left(1+a^{2}-2 a \cos 2(\theta+\varphi-\Phi)\right)^{1 / 2}} .
\end{aligned}
$$

The boundary conditions at the disks can be expressed in dimensionless form as

$$
\begin{aligned}
& F(z=-\lambda, \theta)=\frac{\left(1-a^{2}\right)}{\tau_{\mathrm{R}}\left(1+a^{2}-2 a \cos 2(\theta+\varphi)\right)^{1 / 2}}, \\
& F(z=\Lambda, \theta)=\frac{\left(1-a^{2}\right)}{\tau_{\mathrm{R}}\left(1+a^{2}-2 a \cos 2(\theta+\varphi-\Phi)\right)^{1 / 2}}
\end{aligned}
$$

being

$$
\tau_{\mathrm{R}}=\frac{1}{2 \pi} \int_{0}^{2 \pi} \frac{\left(1-a^{2}\right)}{\left(1+a^{2}-2 a \cos 2 \theta\right)^{1 / 2}} \mathrm{~d} \theta
$$

$$
M(F)=\frac{F\left[1+\left(F_{z}\right)^{2}\right]\left(F_{\theta \theta}-F\right)+F F_{z z}\left[F^{2}+\left(F_{\theta}\right)^{2}\right]-2 F_{\theta}\left(F_{\theta}+F F_{z} F_{z \theta}\right)}{\left\{F^{2}\left[1+\left(F_{z}\right)^{2}\right]+\left(F_{\theta}\right)^{2}\right\}^{3 / 2}}
$$


whereas the azimuthal periodicity condition and the volume preservation condition are

$F(z, \theta+2 \pi)=F(z, \theta)$,

$\frac{1}{2} \int_{-A}^{A} \mathrm{~d} z \int_{0}^{2 \pi} F^{2} \mathrm{~d} \theta=2 \pi A V$

In the above expressions $V$ is the dimensionless volume, $V=\tilde{V} /\left(\pi R^{2} L\right)$, and $A=L /(2 R)$ is the slenderness. Although the numerical analysis allows to consider any volume, in the subsequent sections the volume of the liquid bridge will be fixed to that of the right cylinder of the same slenderness and radii equal to that of the equivalent circular supporting disk, which means $V=1$.

\section{Numerical method}

An algorithm, based on a continuation method (Keller, 1987) capable of over-passing bifurcation points and turning points was developed using a finite-difference method, and was used (Laverón-Simavilla and Perales, 1995; Laverón-Simavilla and Checa, 1997) to obtain the bifurcation diagrams and equilibrium shapes of non-axisymmetric liquid bridges with circular supporting disks subject to non-axisymmetric perturbations such as a lateral acceleration and a misalignment of the disks' axes. In a latter work (Laverón-Simavilla et al., 2003) the stability and equilibrium shapes of a liquid bridge held between a circular disk parallel and coaxial to an elliptic disk subjected to an axial acceleration was studied, it was found that in some cases both effects counteracted in the way of enlarging the stable region in the parameter plane. In this paper, the algorithm is extended to liquid bridges held between elliptic disks in the presence of a lateral acceleration in order to study the influence of the angle formed by the ellipses' main axes, the acceleration direction and the lateral Bond number.

The method is based on linearizing the formulation (Eqs. (3), (5), (6) and (7)) around a known solution $\left(F_{0}(z, \theta), P_{0}\right)$, by seeking solutions of the form

$$
\begin{aligned}
& F(z, \theta)=F_{0}(z, \theta)+f(z, \theta)+o\left(\left|\frac{f}{F_{0}}\right|\right), \\
& P=P_{0}+p+o\left(\left|\frac{p}{P_{0}}\right|\right),
\end{aligned}
$$

where $\left|f 7 F_{0}\right| \ll 1$ and $\left|p / P_{0}\right| \ll 1$. The leading terms obtained from Eq. (3) give an equation for $f(z, \theta)$

$$
\begin{gathered}
\tilde{O}^{-3 / 2}\left\{\tilde{A}+\left(\tilde{B}-\frac{3 \tilde{A} \tilde{Q}}{2 \tilde{O}}\right) f+\left(\tilde{C}-\frac{3 \vec{A} \tilde{S}}{2 \tilde{O}}\right) f_{z}+\left(\tilde{D}-\frac{3 \tilde{A} \tilde{T}}{2 \tilde{O}}\right) f_{\theta}\right\} \\
+\tilde{E} f_{z z}+\tilde{G} f_{\theta \theta}+\tilde{H} f_{z \theta}+P_{0}+p+B_{1}\left(F_{0}+f\right) \cos \theta=0
\end{gathered}
$$

where $\tilde{A}, \vec{B}, \tilde{C}, \tilde{D}, \tilde{E}, \tilde{G}, \tilde{H}, \tilde{O}, \tilde{Q}, \tilde{S}$, and $\tilde{T}$ are known functions of $F_{0}(z, \theta)$ and consequently of the point considered on the interface.
The leading terms obtained for the boundary conditions and the constraint that determines the reference pressure are

$$
\begin{aligned}
& \int_{-\lambda}^{\lambda} \mathrm{d} z \int_{0}^{2 \pi} F_{0}(z, \theta)^{2} \mathrm{~d} \theta+2 \int_{-A}^{\lambda} \mathrm{d} z \int_{0}^{2 \pi}\left[F_{0}(z, \theta) f(z, \theta)\right] \mathrm{d} \theta \\
& =4 \pi \Lambda
\end{aligned}
$$

$F_{0}(\Lambda, \theta)+f(\Lambda, \theta)-\frac{\left(1-a^{2}\right)}{\tau_{\mathrm{R}}\left(1+a^{2}-2 a \cos 2(\theta+\varphi-\Phi)\right)^{1 / 2}}=0$,

$F_{0}(-\Lambda, \theta)+f(-A, \theta)-\frac{\left(1-a^{2}\right)}{\tau_{\mathrm{R}}\left(1+a^{2}-2 a \cos 2(\theta+\varphi)\right)^{1 / 2}}=0$,

$f(z, \theta)=f(z, \theta+2 \pi)$,

where if $\left(F_{0}(z, \theta), P_{0}\right)$ were an exact solution of the problem, in Eqs. (9)-(13) some terms would cancel each other, but all terms have been retained because $\left(F_{0}(z, \theta), P_{0}\right)$ will only be an approximation to the solution in the iterative scheme.

A finite difference scheme has been developed characterizing the mesh as the intersection between the free surface and the planes

$$
\begin{aligned}
& z=A\left(\frac{2 j}{J}-1\right), \quad j=0,1, \ldots, J, \\
& \theta=\frac{2 \pi}{I+1} i, \quad i=0,1, \ldots, I .
\end{aligned}
$$

Doing so, the system (9)-(13) yield a linearized finite-difference equations system which can be written as follows:

$$
\begin{aligned}
& \alpha_{i j} f_{j}^{\prime}+\beta_{i j} f_{j-1}^{i}+\gamma_{i j} f_{j+1}^{\prime}+\delta_{i j} f_{j}^{i-1}+\varphi_{i j} f_{j}^{+1} \\
& \quad+\phi_{i j}\left(f_{j+1}^{i+1}-f_{j+1}^{\prime-1}-f_{j-1}^{++1}+f_{j-1}^{i-1}\right)+p=\psi_{i j}, \\
& \quad i=0, \ldots, I, j=0, \ldots, J, \\
& f_{J}^{i}=\frac{\left(1-a^{2}\right)}{\tau_{\mathrm{R}}\left(1+a^{2}-2 a \cos 2\left(\theta_{i}+\varphi-\Phi\right)\right)^{1 / 2}}-F_{0, j}^{\prime}, \quad i=1, \ldots, I,
\end{aligned}
$$

$f_{0}^{i}=\frac{\left(1-a^{2}\right)}{\tau_{\mathrm{R}}\left(1+a^{2}-2 a \cos 2\left(\theta_{1}+\varphi\right)\right)^{1 / 2}}-F_{0,0}^{i}, \quad i=1, \ldots, I$,

$\sum_{j=0}^{I} \sum_{j=0}^{J} a_{i j} f_{j}^{i}=A$

$f_{j}^{0}-f_{j}^{I+1}=0$,

where the coefficients $\alpha_{i j}, \beta_{i j}, \gamma_{i j}, \delta_{i j}, \varphi_{i j}, \phi_{i j}, \psi_{i j}, a_{i j}$ and $A$ are functions of the values $F_{0, j}^{t}$, and $P_{0}$. 


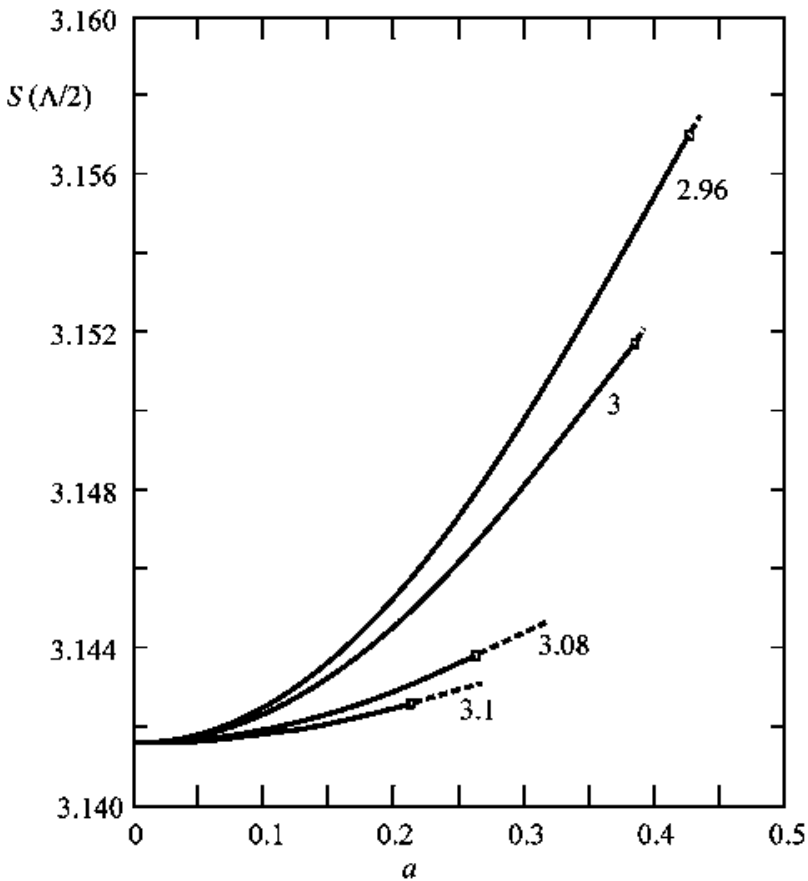

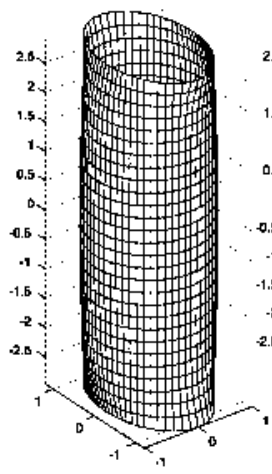

(a)

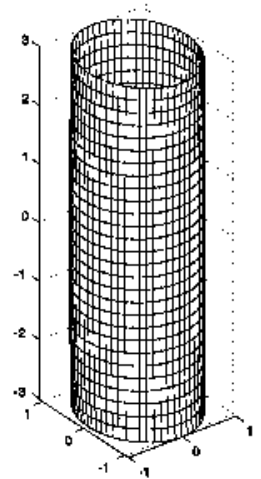

(d)

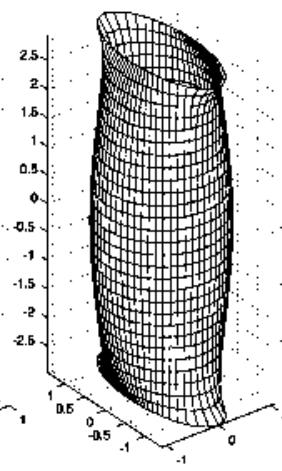

(b)

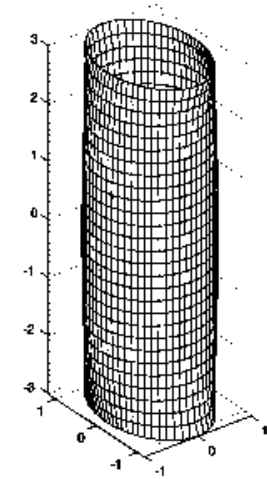

(e)

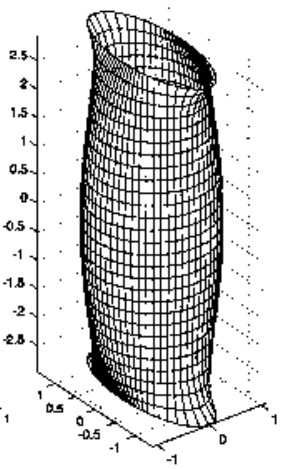

(c)
Fig. 2. Bifurcation diagram for four liquid bridges with slenderness $A=2.96,3.0,3.08$ and 3.1. The area of section $z=A / 2$ is represented versus the parameter measuring the eccentricity of the supporting disks. Stable branches are plotted with solid lines, whilst unstable branches are represented with dashed lines. The bilurcation points are represented with a square. Equilibrium shapes (a), (b) and (c) correspond to $A=2.96$ and $\mathrm{a}=0.2005,0.4201$ and 0.4326 , respectively. Equilibrium shapes (d), (c) and (f) correspond to $A=3.1$ and $\mathrm{a}=0.0828,0.1762$ and 0.2683 , respectively. Shapes (c) and (f) are unstable.
If no further modifications were made, the algorithm would destabilize when crossing any critical point (both turning points and pitch-fork points). To stabilize the algorithm, a new equation needs to be included. With the method modified in this way a sequence of equilibrium shapes is obtained whether they are stable or unstable. The details of the numerical methods used to locate bifurcation and limit points in the families of equilibrium shapes are identical to those outlined elsewhere (Laverón-Simavilla and Perales, 1995) and will not be repeated here.

The extra equation added to stabilize the method defines the arc-length parameter. Two different arc-length parameters are defined, one to evolve in the parameter measuring the eccentricity of the supporting disks, $a$, and another one to evolve in the lateral Bond number, $B_{1}$. In both cases the arc's origin necds to be periodically actualized because of the crror introduced with the discretization. To define the are's origin a second known configuration of the family is needed, the procedure used in each case studied is explained in detail in the following section. The integration must therefore always start from two known configurations in order to define the starting point and the advancing direction along the bifurcation diagram. It is also important to start the integration with known stable shapes because the numerical method can determine a change in the stability character but not the stability character itself. If starting from a stable cquilibrium shape, a parameter can be increased (or decreased) to obtain a scquence of stable equilibrium shapes until a bifurcation or a turning point is encountered.

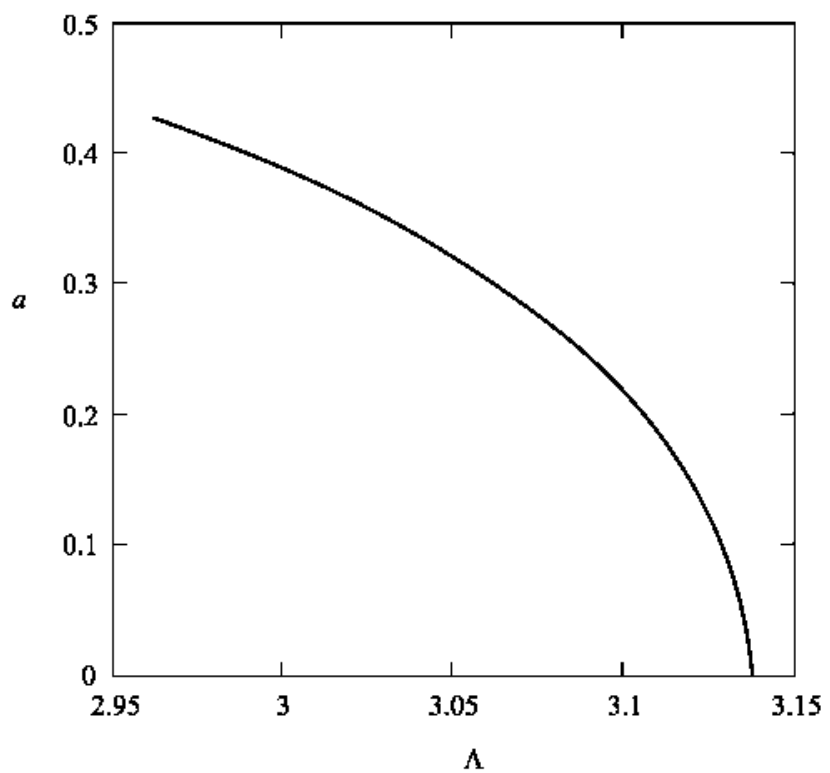

Fig. 3. Stability limit of a liquid bridge held between equal, coaxial and parallel elliptic disks, being $a$ the parameter measuring the eccentricity of the ellipse. 


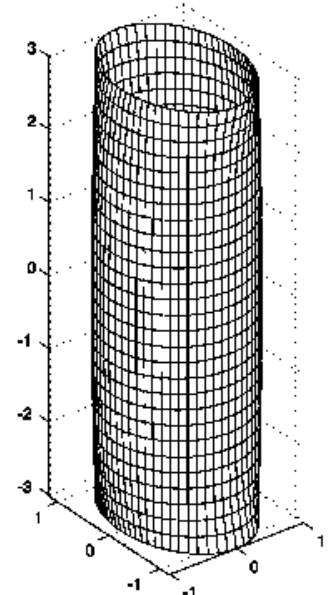

(a)

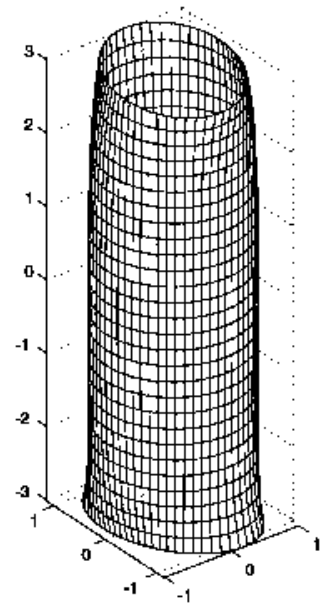

(d)

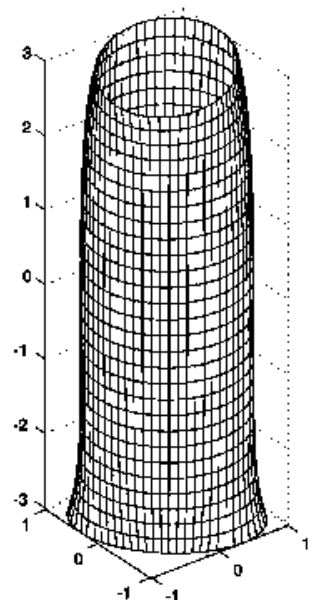

(g)

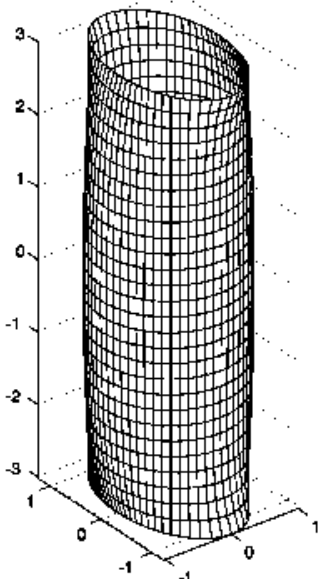

(b)

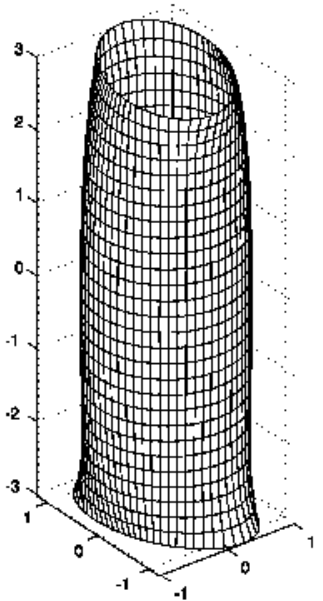

(e)

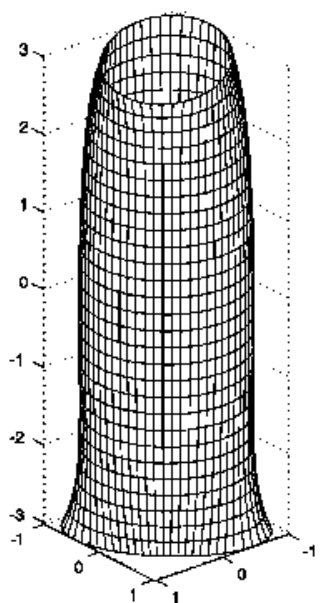

(h)

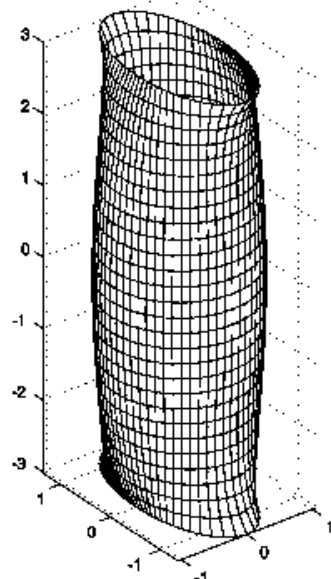

(c)

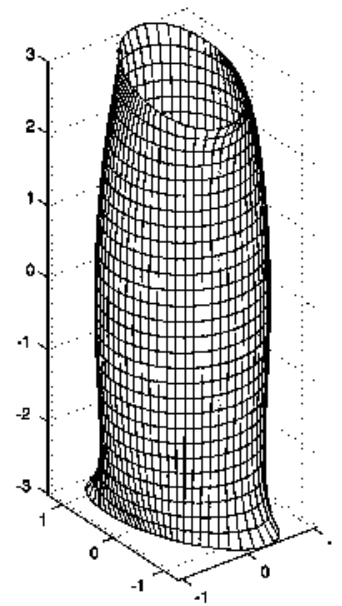

(f)

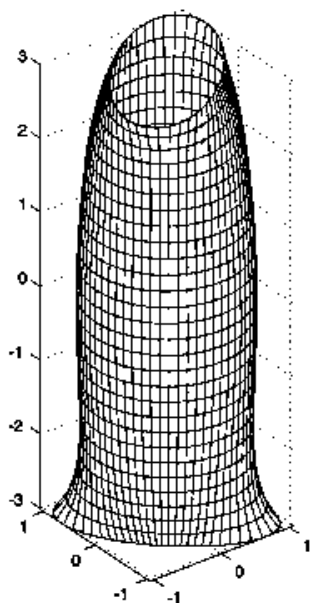

(i)

Fig. 4. Influence of the twist of the supporting disks in the equilibrium shapes. All the configurations correspond to $A=3.02$. Equilibrium shapes (a), (b) and (c) correspond to $\Phi=0$ and $a=1.669,0.2355$ and 0.3421 , respectively. Equilibrium shapes (d), (e) and ( $)$ correspond to $\Phi=\pi / 5$ and $\mathrm{a}=1.670,0.2355$ and 0.3421 , respectively. Equilibrium shapes $(\mathrm{g})$, (h) and (i) correspond to $\Phi=\pi / 2$ and $\mathrm{a}=1.674,0.2359$ and 0.3425 , respectivcly. 


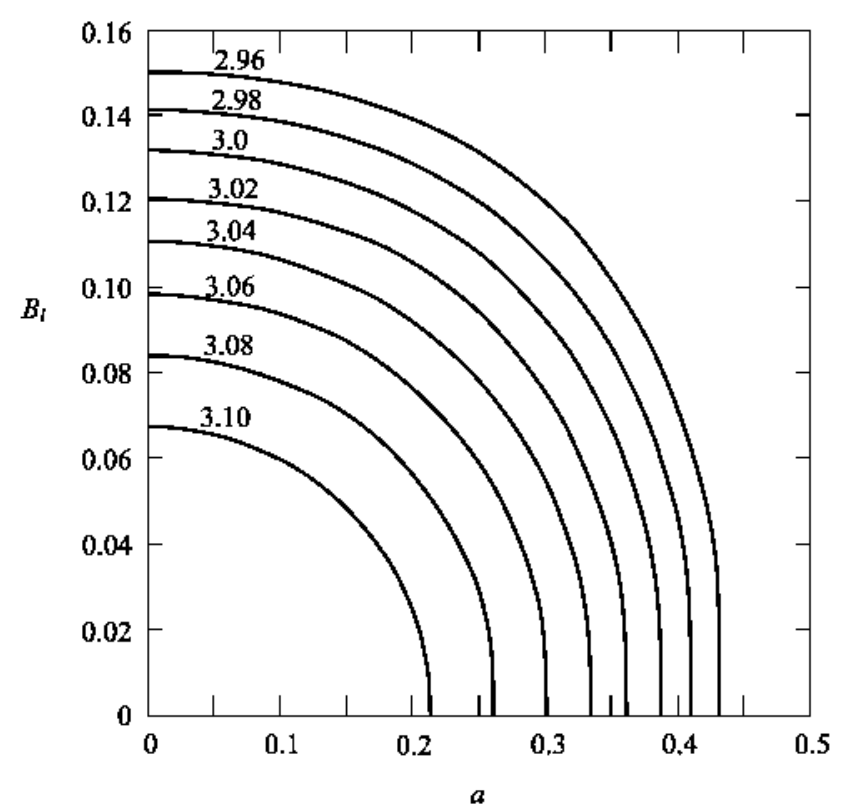

Fig. 5. Maximum values of the lateral Bond number, $B_{1}$, and the parameter measuring the eccentricity, $a$, that liquid bridges of dillerent slenderness can withstand.

\section{Results}

In the following the influence of the eccentricity of the ellipses, the angle formed by their bigger axes, and the lateral acceleration are analyzed.

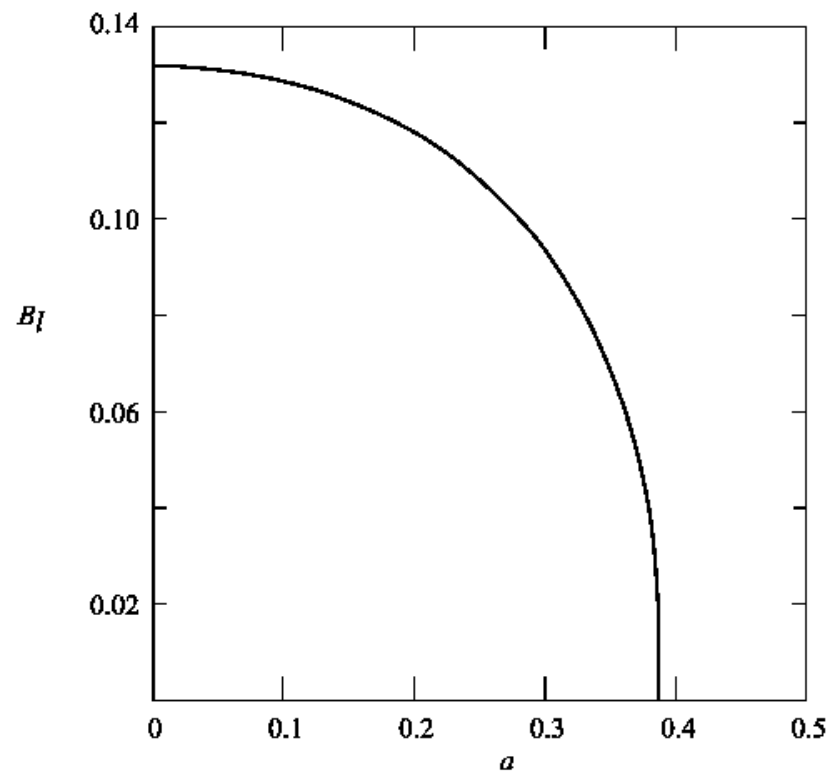

Fig. 6. Maximum values of the lateral Bond number, $B_{1}$, and the parameter measuring the ccentricity, $a$, that liquid bridges with $A=3.0$ and $\Phi=\pi /, 19 \pi / 20,3 \pi / 5,3 \pi / 10, \pi / 5$ and $\pi / 10$ can withstand. All curves collapse in a single one within the numerical method accuracy.
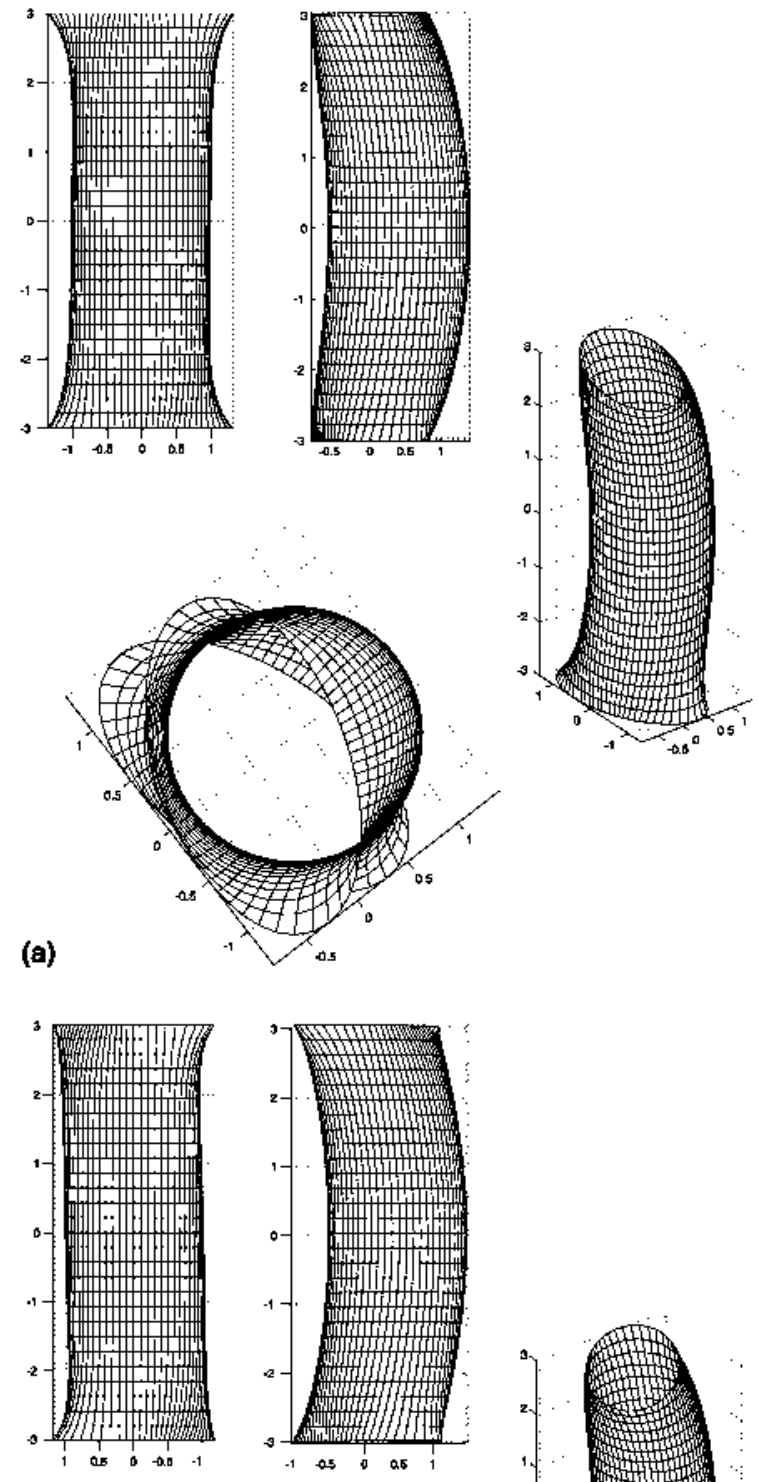

(b)

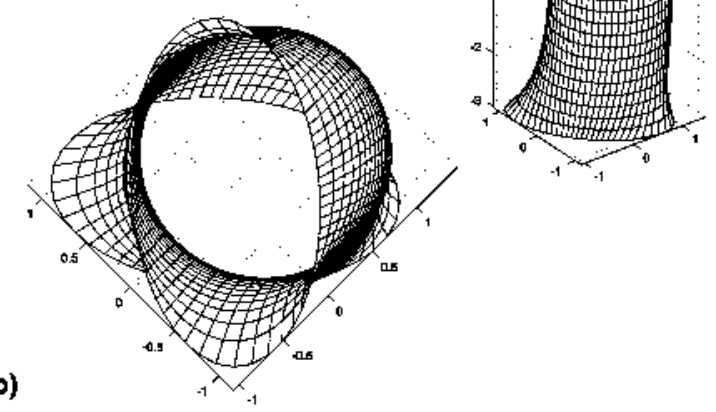

Fig. 7. Three perpendicular views of two stable equilibrium shapes of a liquid bridge with $A=3.0$, (a) $a=0.2957, B_{1}=0.0952, \Phi=4 \pi / 5$, (b) $a=0.2993, B_{1}=0.0943, \Phi=11 \pi / 20$.

\subsection{Absence of lateral acceleration}

It is well known that there is a critical slenderness for cylindrical liquid bridges for which a bifurcation is 
encountered. That is the slenderness corresponding to the minimum volume stability limit. For values of the slenderness greater than the critical the liquid bridges are unstable. The same type of bifurcation exists for liquid bridges held between elliptic supporting disks, being the value of the critical slenderness smaller as the parameter $a$, which accounts for the ellipse eccentricity of the disks, increases.

The main branch of the bifurcation diagram obtained by representing the area of section $z=\Lambda / 2$ as a function of the parameter used to measure the ellipse eccentricity, $a$, is shown in Fig. 2 for several values of the liquid bridge slenderness. The stable branch is represented with a solid line, the bifurcation point is represented with a square and the unstable branch is represented with a dashed line. The equilibrium shapes corresponding to $A=3.1, A=2.96$ and three different values of the parameter measuring the eccentricity of the supporting disks are also represented in the figure, the first two equilibrium shapes for cach slenderness are stable configurations, whilst the others are unstable. It can be seen from the figure how short liquid bridges can withstand greater deformations without breaking. Fig. 3 shows the relation between the critical slenderness and the parameter measuring the eccentricity of the ellipses. Although there is an asymptotic study done for

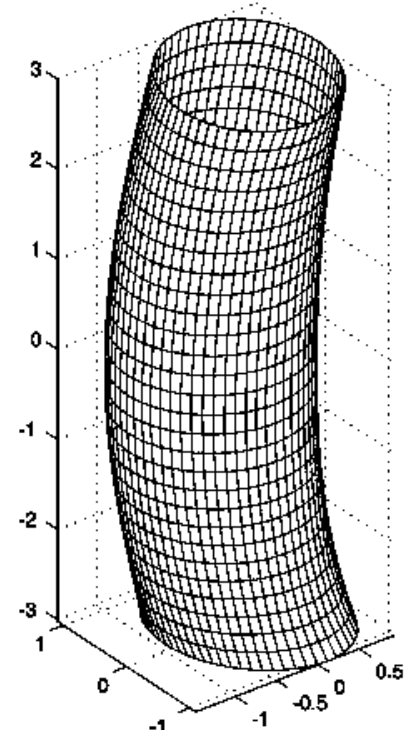

(a)

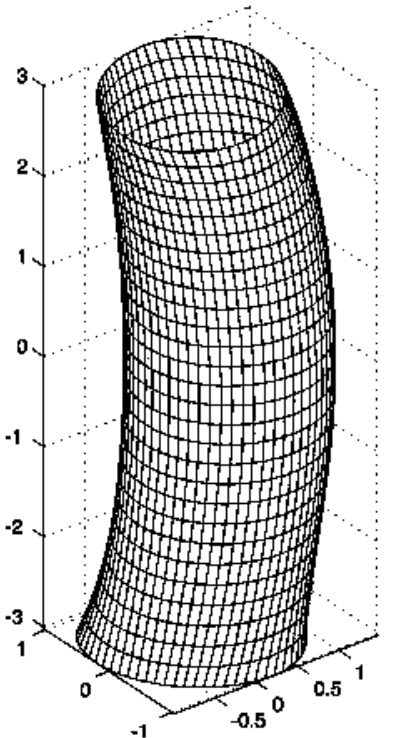

(d)

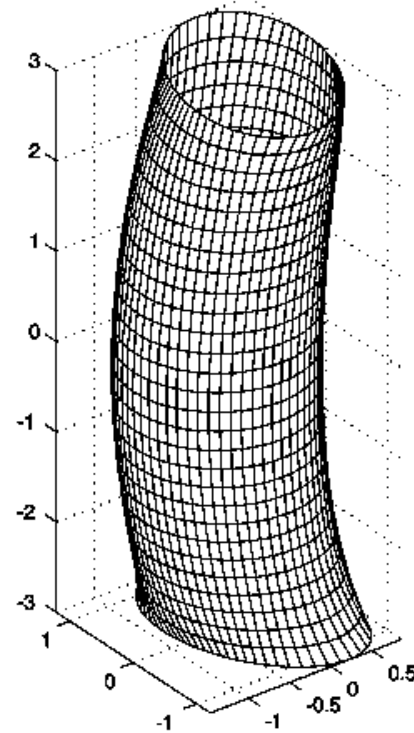

(b)

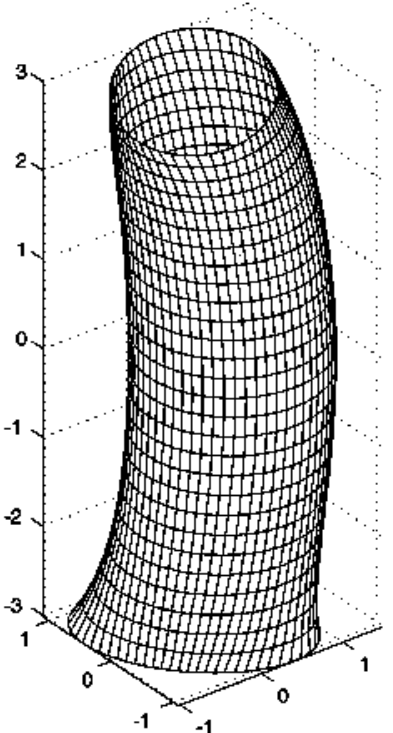

(e)

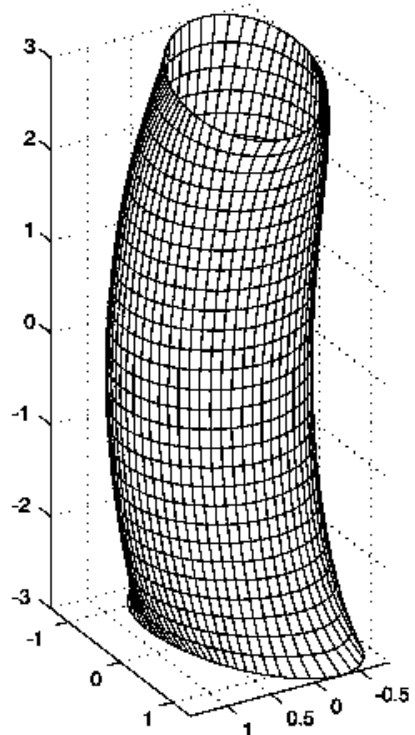

(c)

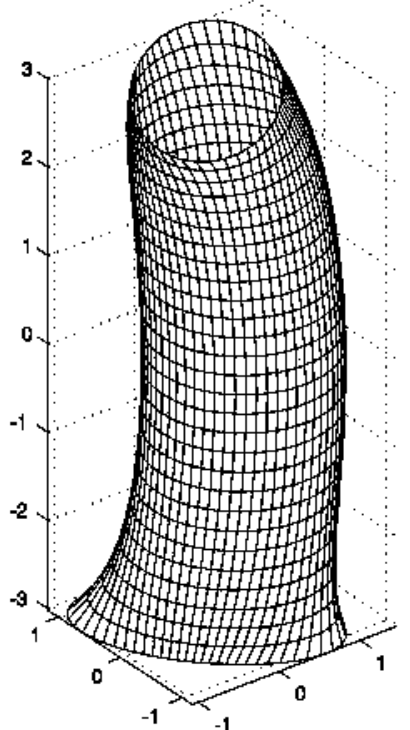

(f)

Fig. 8. Stable equilibrium shapes of liquid bridges with $A=3.0$. The first three shapes correspond to $\Phi=4 \pi / 5$, (a) $a=0.1015, B_{1}=0.1016$, (b) $a=0.2134, B_{1}=0.1012$, (c) $a=0.2957, B_{1}=0.0952$; and the last three correspond to $\Phi=11 \pi / 20$ and (d) $a=0.0997, B_{1}=0.0989$, (e) $a=0.2041$, $B_{1}=0.0995,\left(\right.$ ) $a=0.2993, B_{1}=0.0943$. 


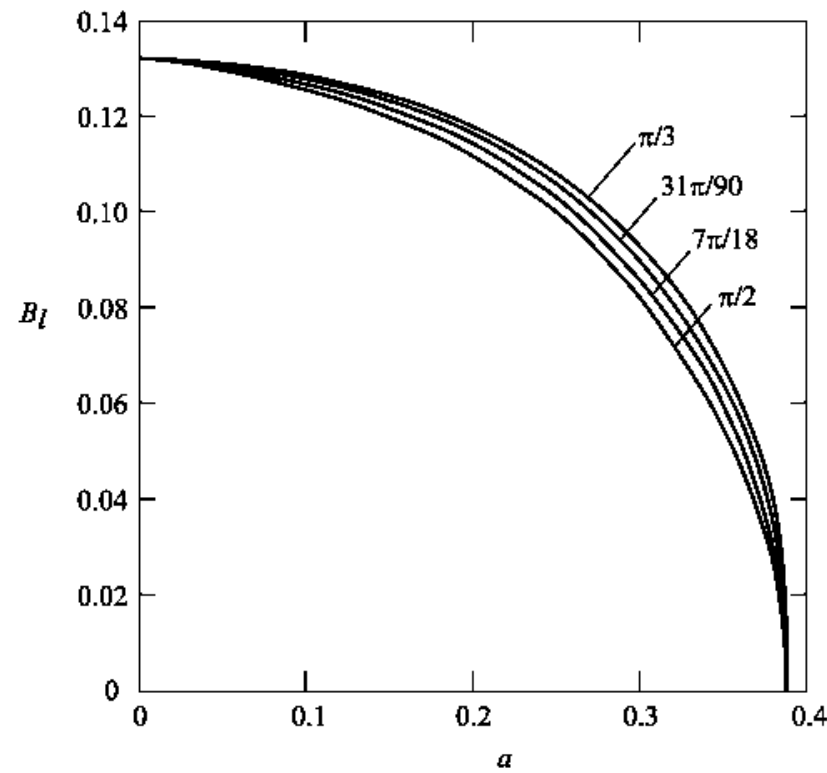

Fig. 9. Maximum values of the lateral Bond number, $B_{1}$, and the parameter measuring the eccentricity, $a$, that liquid bridges with $A=3.0$ and $\Phi=2 \pi / 3$ and $\varphi=\pi / 2,7 \pi / 18,31 \pi / 90$ and $\pi / 3$ can withstand.

non-axysimmetric supporting disks (Meseguer et al., 2001) the results here obtained cannot be compared to the analytical ones because only the first order effect was retained in the paper, the term retained there is related to the difference in area of the supporting disks and not really to their shape.

The equilibrium shapes and stability limits have also been calculated varying the angle formed by the main axes of the supporting disks, $\Phi$, but no significant differences where found in the values of the critical slenderness varying this parameter. Several stable equilibrium shapes are represented in Fig. 4 for different values of $\Phi$.

\subsection{Influence of lateral acceleration}

The influence of a lateral acceleration is here considered, being the angle formed by the principal axis of the supporting disk, $\Phi$, and the angle formed by the lateral acceleration and the main axis of the lower supporting disk, $\varphi$. The equilibrium shape of a stable liquid bridge held between elliptic supporting disks is calculated using a cylindrical initial shape $\left(a=B_{1}=0\right)$ and evolving in the lateral Bond number, $B_{1}$. The initial equilibrium shape for $a \neq 0$ is one calculated evolving in $a$. The second initial equilibrium shape required $\left(B_{1} \simeq 0, a \neq 0\right)$ cannot be calculated from any analytical expression given by an asymptotic analysis, therefore the equilibrium shape calculated for the previous value of $a$, and $B_{1} \simeq 0$ in the previous iteration in $B_{1}$ is used. The evolution is again done in the lateral Bond number, $B_{1}$, until a bifurcation point is encountered.

The stability limits for liquid bridges of different slenderness, $\Phi=0$ and $\varphi=0$ are plotted in Fig. 5. Both per-

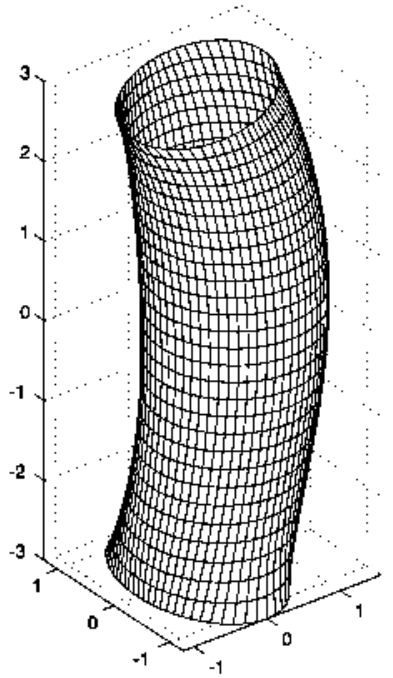

(a)

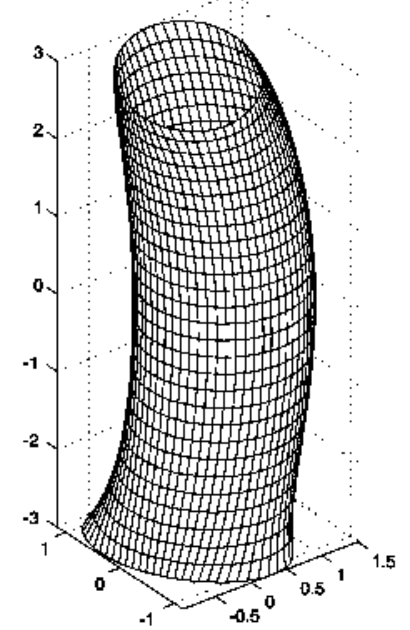

(c)

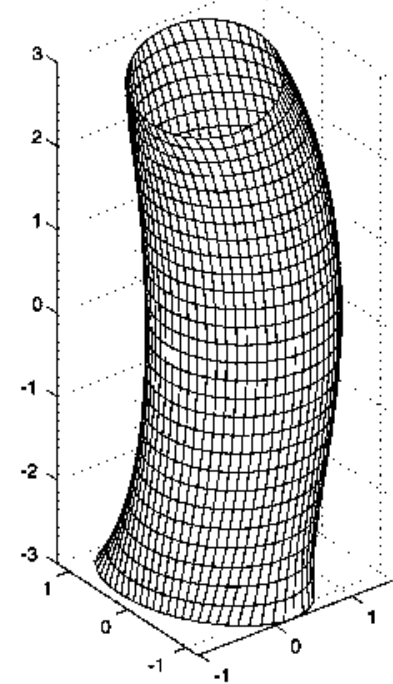

(b)

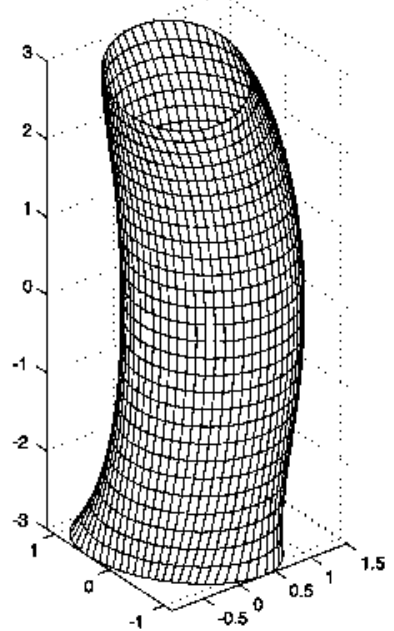

(d)
Fig. 10. Stable equilibrium shapes of four liquid bridges with $A=3.0$, and $\Phi=2 \pi / 3$ and (a) $\varphi=\pi / 2$, (b) $\varphi=7 \pi / 18$, (c) $\varphi=31 \pi / 90$ and (d) $\varphi=\pi / 3$.

turbations, $a$ and $B_{1}$, cause a des-stabilizing effect which reduces the stable region.

In order to study the influence of the twist of the supporting disks, the stability limits have been calculated for a liquid bridge with $A=3.0$, the acceleration pointing in the direction of the bisector of the angle formed by the bigger main axes of the supporting disks ( $\varphi=\Phi / 2)$. In this particular case, it has been found that the stability limit does not depend on the twist of the disks, $\Phi$. All the bifurcation points lie on the same curve irrespective to the value of $\Phi$, as represented in Fig. 6 . Three different vicws of an almost unstable liquid bridge with $A=3.0$ are represented in Fig. 7 for two values of $\Phi$. Several stable equilibrium shapes are represented in Fig. 8, corresponding to two different values of the twist of the liquid bridge and for several values of the parameter measuring the eccentricity of the supporting disks, $a$, and the lateral Bond number, $B_{1}$. 
The effect on the stability limit of changing the direction of the acceleration for a liquid bridge with $A=3.0$ and $\phi=2 \pi / 3$ is represented in Fig. 9. The figure shows that the stable region becomes smaller as the angle between the acceleration and the bigger axes of the lower supporting disk decreases, being minimum for $\varphi=\pi / 2$ and maximum for $\varphi=\Phi / 2$. Four equilibrium shapes corresponding to $\Phi=2 \pi / 3, B_{1}=0.110, a=0.2041$, $A=3.0$ and $\varphi=\pi / 2,7 \pi / 18,31 \pi / 90, \pi / 3$ are represented in Fig. 10.

\section{Conclusions}

To summarize, the combined effect of lateral Bond number, shape and twist of the supporting disks have been analyzed numerically. A particular shape of the disks (being both disks equal) has been considered and the equilibrium shapes and stability limits calculated. It is interesting to observe how the twist of the disks does not affect the stability limit. Breaking the symmetry by changing the direction of the lateral acceleration changes the stability limit. The most stable configuration correspond to the acceleration pointing in the bisector of the angle formed by the bigger main axes of the supporting disks ( $\varphi=\Phi / 2)$, as the liquid bridge is rotated the configuration becomes less stable.

The shape of the sections $z=$ cte of the liquid bridge are nearly circular far from the supporting disks; azimuthal variations due to the non-circularity of the supporting disks are barely present at the middle sections of the liquid bridge. That is the reason why the twist angle of the supporting disks does not change the stability limits in absence of lateral acceleration. The trend to eliminate azimuthal variations when moving away from the supporting disks lead to a narrowing of the liquid bridge in the plane containing the bigger main axis of the nearby supporting disk and a widening in the plane containing the smaller main axis of the disk. Near the supporting disks there is also a neck that gets narrower as the parameter measuring the eccentricity of the disks increases. Therefore the narrowing of the liquid bridge in the direction of the bigger main axes increases as the parameter measuring the disks' eccentricity increases making those directions the most unstable ones.

\section{References}

Gonzalez, H. Castellanos, A. The effect of residual gravity on the stability of liquid columns subjected to electric fields. J. Fluid Mech. 249, 185. 1993.

Keller. H.B. Lectures on Numerical Methods in Bifurcation Problems. Springer-Verlag, Berlin. 1987.

Laveron-Simavilla, A., Checa. E. Effect of a lateral gravitational field on the nonaxisynmetric equilibrium shapes of liquid bridges held between eccentric disks and of volumes equal to those of cylinders. Phys. Fluids 9, 817, 1997.

Laverón-Simavilla. A.. Perales. J.M. Equilibrium shapes of nonaxisymmetric liquid bridges of arbitrary volume in gravitational fields and their potential energy. Phys. Fluids 7, 1204. 1995.

Laverón-Simavilla. A., Meseguer, J., Espino, J.L. Stability of liquid bridges between an elliptical and a circular supporting disk. Phys. Fluids 15. 2830, 2003.

Lowry, B.J., Steen, P.H. Stability of slender liquid bridges subjected to axial flows. J. Fluid Mech. 330. 189, 1997.

Luengo, V. Meseguer, J., Parra, I.E. Experimental study of the stability of long, axisynmetric liquid bridges between solid supports at different temperatures. Exp. Fluids 34. 412, 2003.

Mahajan. M.P., Zhang, S., Tsinge, M., Taylor, P.L., Rosenblatt, C. Stability of magnetically levitated liquid bridges of arbitrary volume subjected to axial and lateral gravity. J. Colloid Interface Sci. 213, 592. 1999

Marr-Lyon, M.J., Thiessen, D.B., Marston, P.L. Stabilization of a cylindrical capillary bridge far beyond the Rayleigh-Platean limit using acoustic radiation pressure and active feedback. J. Fluid Mech. 351, 345. 1997.

Martínez. I., Meseguer, J., Perales. J.M. Analytical modeling of floating zone crystal growth. Adv. Space Res. 29, 569, 2002a.

Martínez, I., Meseguer. J., Perales. J.M. Simulation of nonaxisynmetric floating zone crystal growth under microgravity. Microgravity Sci. Technol. 13/2, 3, 2002b.

Meseguer, J., Perales, J.M., Alexander, J.I.D. A perturbation analysis of the stability of long liquid bridges between almost circular supporting disks. Phys. Fluids 13. 2724. 2001.

Parra, I.E. Perales. J.M.. Meseguer, J. On the influence of Marangoni convection on the stability of liquid bridge interfaces. Adv. Space Res. 29, 625, 2002.

Slobozhanin. L.A.. Alexander, J.I.D. Combined effect of disk inequality and axial gravity on axisymmetric liquid bridge stability. Phys. Fluids 10, 2473, 1998.

Slobozhanin. L.A., Perales. J.M. Stability of liquid bridges between equal disks in an axial gravity field. Phys. Fluids A 5, 1305, 1993. 